This item was submitted to Loughborough's Research Repository by the author.

Items in Figshare are protected by copyright, with all rights reserved, unless otherwise indicated.

\title{
A methodology for the performance evaluation of inertial measurement units
}

PLEASE CITE THE PUBLISHED VERSION

http://dx.doi.org/10.1007/s10846-012-9772-8

\section{PUBLISHER}

(C) Springer

\section{VERSION}

AM (Accepted Manuscript)

\section{PUBLISHER STATEMENT}

This work is made available according to the conditions of the Creative Commons Attribution-NonCommercialNoDerivatives 4.0 International (CC BY-NC-ND 4.0) licence. Full details of this licence are available at: https://creativecommons.org/licenses/by-nc-nd/4.0/

\section{LICENCE}

CC BY-NC-ND 4.0

\section{REPOSITORY RECORD}

Sessa, Salvatore, Massimiliano Zecca, Zhuohua Lin, Luca Bartolomeo, Hiroyuki Ishii, and Atsuo Takanishi. 2019. "A Methodology for the Performance Evaluation of Inertial Measurement Units". figshare.

https://hdl.handle.net/2134/19373. 


\title{
A methodology for the performance evaluation of Inertial Measurement Units
}

Salvatore Sessa*, ${ }^{*}$, Massimiliano Zecca ${ }^{2,3,4}$, Zhuohua Lin ${ }^{1,5}$, Luca Bartolomeo ${ }^{1,5}$, Hiroyuki Ishii ${ }^{6}$, and Atsuo Takanishi ${ }^{2,3,4,5}$

${ }^{1}$ Faculty of Science and Engineering, Graduate School of Creative Science and Engineering, Waseda University.

${ }^{2}$ Faculty of Science and Engineering, School of Creative Science and Engineering, Waseda University.

${ }^{3}$ HRI - Humanoid Robotics Institute, Waseda University

${ }^{4}$ Italy-Japan Joint Laboratory on Humanoid and Personal Robotics

"RoboCasa", Waseda University.

${ }^{5}$ Global Robot Academia, Waseda University.

${ }^{6}$ Faculty of Science and Engineering, Waseda Research Institute for Science and Engineering, Waseda University.

(*) Corresponding author

E-mail: contact@takanishi.mech.waseda.ac.jp

Address: 2-2 Wakamatsu-cho, Shinjuku-ku

Tokyo 168-8480, JAPAN

TWIns - Room 03 C-301

Phone: +81-3-5369-7369 ext 3604

Fax: $+81-3-5269-9061$

URL: http://www.takanishi.mech.waseda.ac.jp

\begin{abstract}
:
This paper presents a methodology for a reliable comparison among Inertial Measurement Units or attitude estimation devices in a Vicon environment. The misalignment among the reference systems and the lack of synchronization among the devices are the main problems for the correct performance evaluation using Vicon as reference measurement system. We propose a genetic algorithm coupled with Dynamic Time Warping (DTW) to solve these issues. To validate the efficacy of the methodology, a performance comparison is implemented between the WB-3 ultraminiaturized Inertial Measurement Unit (IMU), developed by our group, with the commercial IMU InertiaCube ${ }^{\text {TM }}$ by InterSense.
\end{abstract}

Keywords: Performance evaluation, Inertial Measurement Units, Motion Capture Systems, Motion Sensors Calibration. 


\section{Introduction}

In the last years, the interest on motion capture systems has increased in many application fields. Medical applications, for example, paid a growing attention to human motion caption systems to obtain more efficient rehabilitation therapies [1], [2], and to build monitoring networks for patients and elderly people in the hospitals and in their own homes [3], [4]. In the game industry, the market trend is toward active game platforms, where a smart wearable motion capture suit could expand the capability of this kind of games and open total virtual reality scenarios, at the edge of the augmented reality [5], [6].

The human body can be modeled as a set of links, or limb segments, arranged in a tree-like structure in which each individual limb segment can be treated as rigid body. Specifying the posture of this model involves the descriptions of the orientation and position of each segment [7]. The nature of the relationships between the links in the structure determines whether the positions and orientations of the segments are described individually or they are specified in relation to the others. The formalisms chosen have the following important effects: ability to represent orientations without problems of singularity; computational efficiency; storage requirements; and transmission bandwidth requirements.

Several motion capture technologies have been developed in the past years, such as optical, image-based [8], mechanical [9], magnetic[10], [11], and acoustic [12]. In particular, the optical motion capture systems are preferred in the computeranimation community, in the film industry and in medical contexts [13], [14], because they offer a reliable and accurate way to record the motions of complex systems. However, this approach is expensive and poor in portability. These systems compute the exact locations of markers from the images recorded by the surrounding cameras using triangulation methods; therefore, they can be used only in a structured environment [15]. Furthermore, the markers must be always in the field of view of the camera to have reliable measurements.

A very promising frontier on the wearable and reliable Motion Capture system is based on Inertial Measurement Unit (IMU) that can be virtually used everywhere. 
Many studies of human motion tracking using these inertial sensors have been performed [16-19]. A simple approach to inertial orientation tracking might involve the integration of angular rate data to determine the attitude. However, this solution would be prone to drift over time due to bias and drift errors. In order to avoid the drift, inertial tracking systems make use of additional complementary sensors. Commonly, these sensors include triads of accelerometers and magnetometers for referencing respectively the gravity and the magnetic field vectors. The use of accelerometers allows the estimation of the attitude in respect to the horizontal plane, measuring the gravity vector in the sensor coordinate frame. However, when the sensor module is rotated around the vertical axis, the projection of the gravity vector on each of the principal axes of the accelerometer triad will not change. Magnetometers are used to measure the local magnetic field vector in the sensor coordinates, and allow the determination of orientation relative to the vertical rotation.

Data from these incorporated sensors are normally integrated by different sensor fusion algorithms to estimate the orientation, such as Kalman filter, particle filter and complementary filtering algorithms [20-24]. Among these algorithms, the Extended Kalman Filter (EKF) is the most used because of its high accuracy on the attitude estimation and model simplicity [25]. Additionally, the computational efficiency of the EKF is suitable for real time applications [26].

Most of the commercial IMUs do not explain the detail of the attitude estimation algorithm implemented and it is quite difficult to compare the performance among them. The embedded sensors specifications on the attitude evaluation often are not clearly stated and the performances among them are hardly comparable. Additionally, there is the necessity to find a reliable method for the comparison among different types of motion capture systems that are not necessarily synchronized [27]. Therefore, this paper aims at presenting a methodology for a reliable comparison among Inertial Measurement Units, or other devices for the attitude measurement, in a Vicon environment as reference truth [15]. A genetic algorithm coupled with the Dynamic Time Warping (DTW) allowed us to correctly synchronize the data series and compare the attitude obtained by different measurement systems. 
The proposed methodology is applied to the WB-3 Ultra-miniaturized IMU [28], developed by our group, with the commercial IMU InertiaCube $3^{\text {TM }}$ by InterSense [29], considering the Vicon motion capture system [15] as ground truth.

The Section II of the paper, titled "Materials", describes the devices used for the verification of the comparison methodology and the steps to make the data homogeneous. Firstly, the Vicon system and the marker position are described in details. Following, the WB-3 sensors and the algorithm implemented for retrieving the attitude are explained. The WB-3 IMU algorithm is a modification of the quaternion based EKF based on the estimation of the measurement covariance matrix (R-Adaptative Algorithm) [30]. Finally, the commercial InertiaCube $3^{\mathrm{TM}}$ is presented focusing on its main characteristics.

Section III, titled "Methods" focuses on the main idea of the paper. A general method for the reliable comparison of the data acquired from different sensors is described.

Section IV, titled "Experimental Validation", describes the experiments conducted and discusses the results obtained applying the proposed methodology for the comparison.

Finally, Section V summarizes the results obtained and proposes possible application for the proposed methodology in the robotics field.

\section{Materials}

\subsection{Vicon}

Optoelectronics systems that enable three-dimensional analysis of human movements with automatic digitizing and data processing have vastly reduced the time needed to gain results on the human motion analysis. A Vicon motion system uses high-resolution digital strobe technology cameras, which are interfaced directly with a microcomputer, to enable the light to be reflected from "passive markers" on the subject into the camera. The markers are spherical or hemispherical, and covered in retro reflective paper. Through pattern recognition, the shape of the detected light source is compared with the expected shape. The Vicon 612, used in our experiments, has powerful and automated software that allows eight cameras to be used contemporarily. The VCam cameras has selectable frame rate $100 / 119.88 / 150 / 200 \mathrm{~Hz}$ and a resolution of $648 \times 493$ pixels. 
Data are processed in pipeline enabling the acquisition, the elaboration, and the data storage in a single step. Vicon system can reach accuracy level of less than $\pm 0.1 \mathrm{~mm}$. Still, the accuracy depends on many factors, e.g. number of cameras, camera placement, markers placement. The accuracy of the reconstructed 3D position of a marker also depends on the operator that performs the system calibration. A good calibration increases the quality of the linear transformation of a marker's 2D coordinates on the video to its 3D location in the space [31].

An L-frame with four passive markers, which is used also for the static calibration, was installed on a tripod for determine the attitude on the space of the object inside the Vicon environment (Figure 1). On the L-Frame, we fixed also the WB-3 IMU and the Intersense sensor (Figure 2) that will be described in detail on the following sections.

The markers $\mathrm{V}_{0}, \mathrm{~V}_{2}$ and $\mathrm{V}_{3}$ are used for the attitude calculation, the marker $\mathrm{V}_{1}$ was used to clearly differentiate between $V_{2}$ and $V_{3}$ during the experiment.

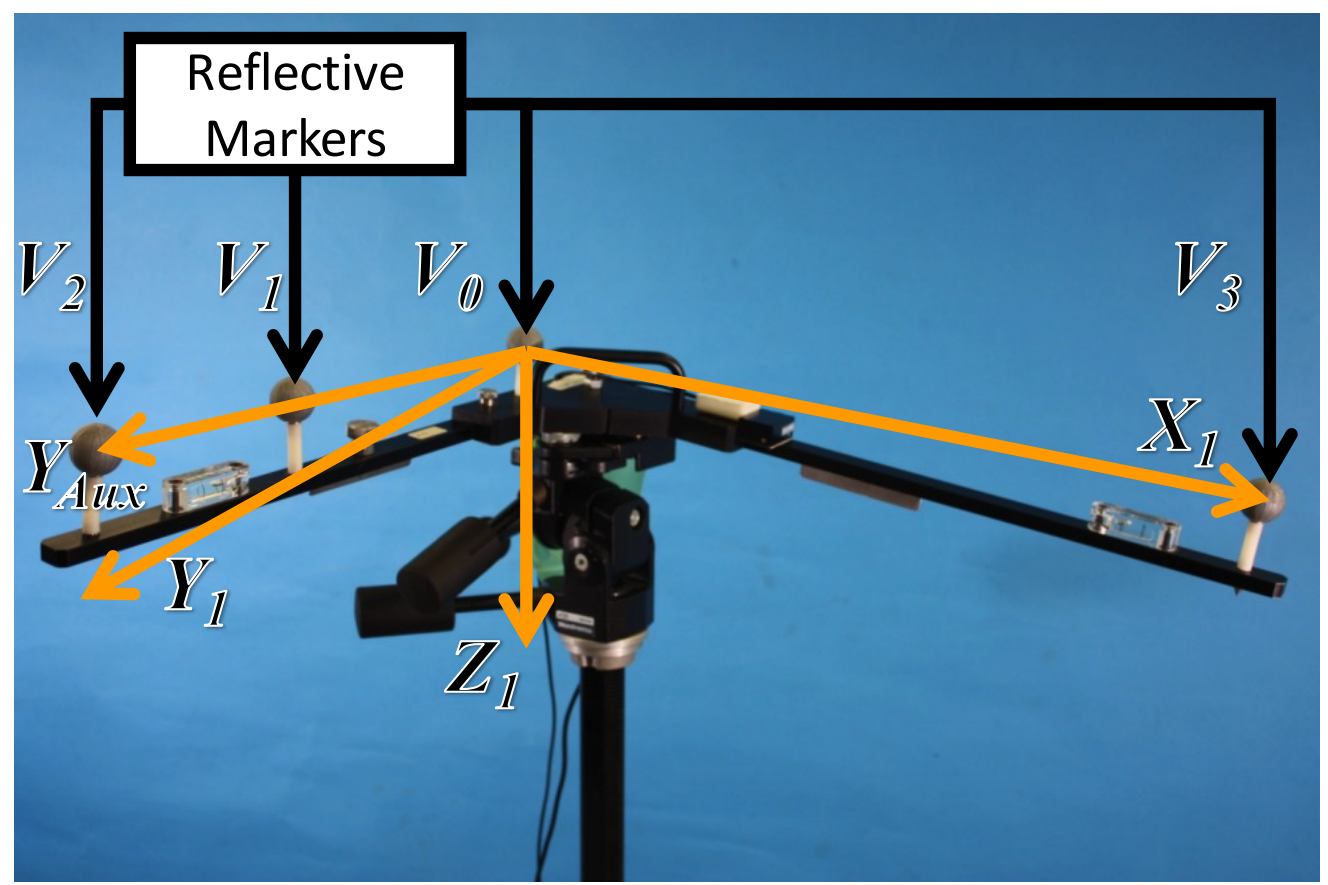

Figure 1: L-frame with reflective markers, InertiaCube ${ }^{\mathrm{TM}}$ and WB-3. 


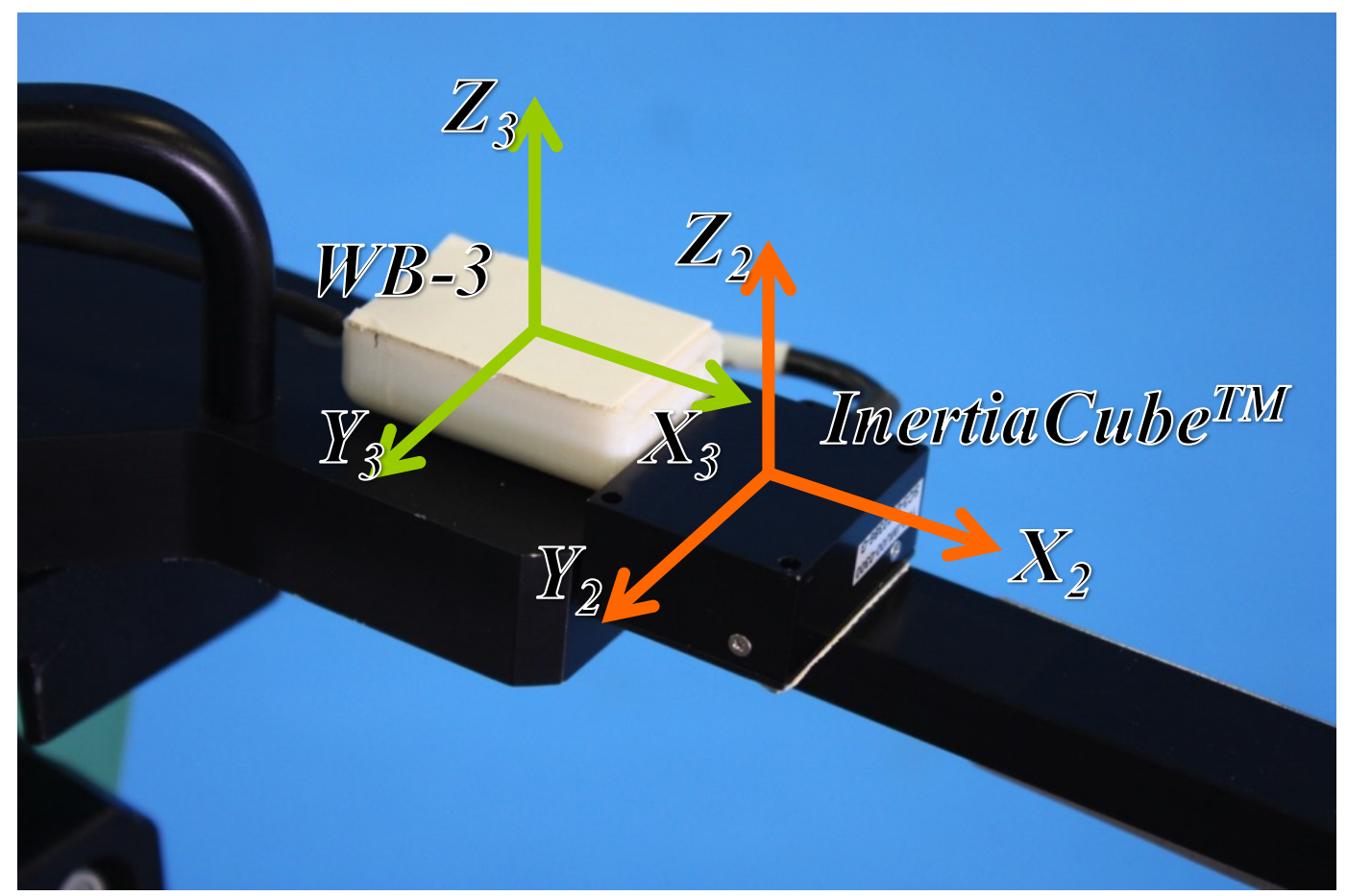

Figure 2: WB-3 and InertiaCube $3^{\mathrm{TM}}$ detail.

If $\left[V_{i_{x}}, V_{i_{v}}, V_{i_{z}}\right] i=0 \ldots 3$ indicates the coordinates of the marker $V_{i}$ in the Vicon reference frame, then the direction cosine of the frame $\left(\hat{X}_{1}, \hat{Y}_{1}\right.$, and $\left.\hat{Z}_{1}\right)$ in respect to the Vicon reference system can be calculated as follows:

$$
\begin{aligned}
& \hat{X}_{1}=\left[V_{3_{x}}-V_{0_{x}}, V_{3_{y}}-V_{0_{y}}, V_{3_{z}}-V_{0_{z}}\right]\left\|V_{3_{x}}-V_{0_{x}}, V_{3_{y}}-V_{0_{y}}, V_{3_{z}}-V_{0_{z}}\right\|^{-1} \\
& \hat{Y}_{A u x}=\left[V_{2_{x}}-V_{0_{x}}, V_{2_{y}}-V_{0_{y}}, V_{2_{z}}-V_{0_{z}}\right]\left\|V_{2_{x}}-V_{0_{x}}, V_{2_{y}}-V_{0_{y}}, V_{2_{z}}-V_{0_{z}}\right\|^{-1} \\
& \hat{Z}_{1}=\hat{X}_{1} \times \hat{Y}_{A u x} \\
& \hat{Y}_{1}=\hat{Z}_{1} \times \hat{X}_{1}
\end{aligned}
$$

The rotation matrix of the $\mathrm{L}$ frame in the Vicon reference system is the follow:

$$
M=\left[\hat{X}_{1}, \hat{Y}_{1}, \hat{Z}_{1}\right]^{T}=\left[\begin{array}{lll}
M_{11} & M_{12} & M_{13} \\
M_{21} & M_{22} & M_{23} \\
M_{31} & M_{32} & M_{33}
\end{array}\right]
$$

After, we define the quaternion ${ }_{V I}^{L F} q_{V I}={ }_{V I}^{L F}\left[q_{V I_{0}}, q_{V I_{1}}, q_{V I_{2}}, q_{V I_{3}}\right]$ as the attitude of the L-Frame ( $L F$ leading superscript) measured by the Vicon (VI subscript) in the reference system of the Vicon (VI leading subscript) [32]. ${ }_{V I}^{L F} q_{V I}$ can be computed using the conversion equations from rotation matrix to quaternion. 


$$
\begin{aligned}
& q_{V I_{0}}=\frac{1}{2} \sqrt{1+M_{11}+M_{22}+M_{33}} \\
& q_{V I_{1}}=\frac{1}{4 q_{V I_{0}}}\left(M_{23}-M_{32}\right) \\
& q_{V I_{2}}=\frac{1}{4 q_{V I_{0}}}\left(M_{31}-M_{13}\right) \\
& q_{V I_{3}}=\frac{1}{4 q_{V I_{0}}}\left(M_{12}-M_{21}\right)
\end{aligned}
$$

\section{$2.2 \quad$ WB-3 Inertial Measurement Unit}

Our group developed an ultra-miniaturized IMU named WB-3, which is very compact and lightweight (size $26 \times 20 \times 8 \mathrm{~mm}$ and weight $2.9 \mathrm{~g}$ without housing; size $27 \times 23 \times 11 \mathrm{~mm}$ and weight $6 \mathrm{~g}$ with housing). A picture of the IMU is showed in Figure 3. The communication of WB-3 with the $\mathrm{PC}$ is performed using the CAN Bus interface at $1 \mathrm{Mb} / \mathrm{s}$. The firmware of the microcontroller can be updated by using the JTAG interface. One user LED is employed on the IMU for operating mode indication. The configuration block diagram of the WB-3 IMU is showed in Figure 4.

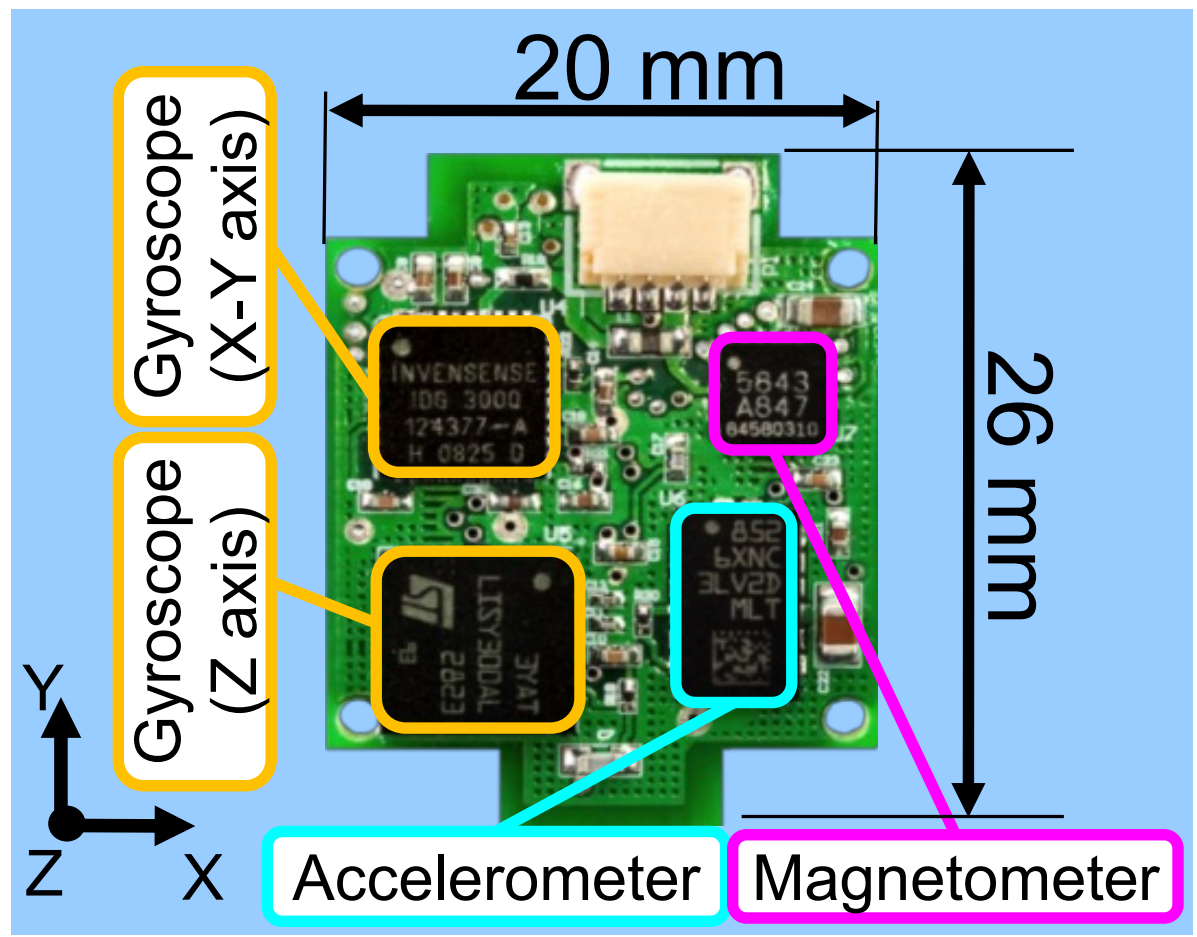

Figure 3: WB-3 Inertial Measurement Unit. 


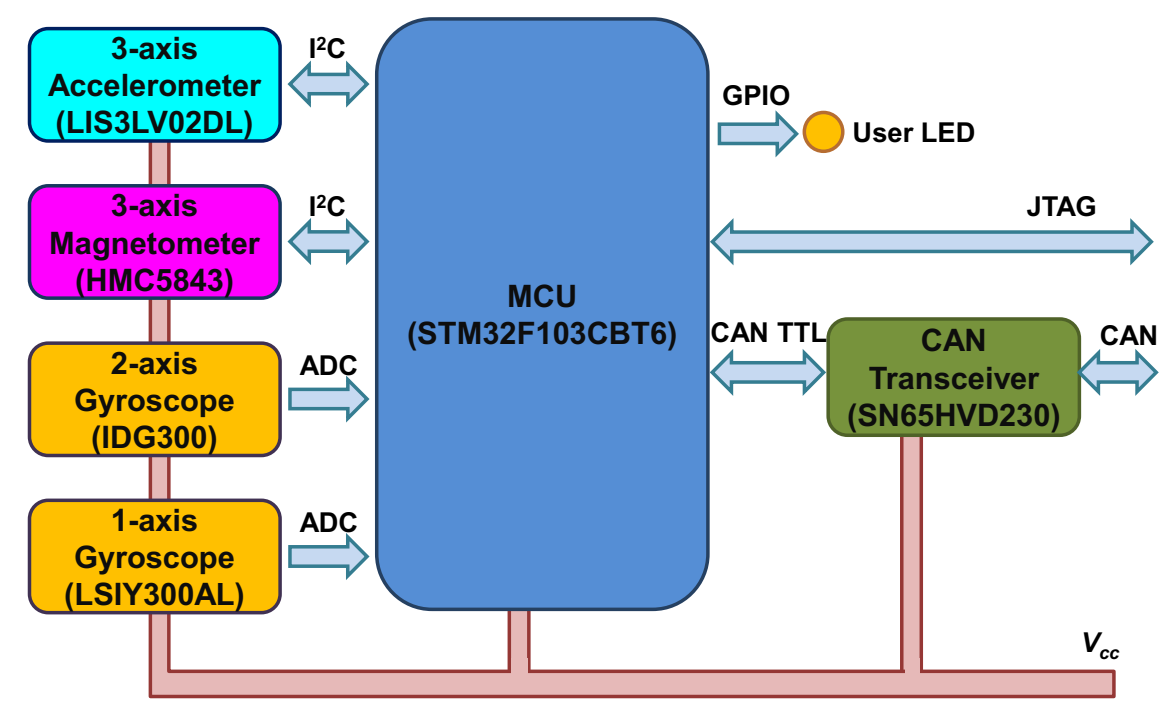

Figure 4: WB-3 block diagram.

The WB-3 is primarily composed of a microcontroller and the following sensors: 3-axis gyroscope, 3-axis accelerometer, and 3-axis magnetometer.

1) Gyroscope: to measure 3-axes angular velocity, a 2-axis gyro IDG300 (InvenSense) and a 1-axis gyro LISY300AL (STMicroelectronics) were employed. The LISY300AL is a miniaturized 7.0x7.0x1.9 mm z-axis gyro sensor. Its full-scale is $\pm 300 \mathrm{Deg} / \mathrm{s}$ with a Bandwidth of $88 \mathrm{~Hz}$ and a sensitivity of $3.3 \mathrm{mV} / \mathrm{Deg} / \mathrm{s}$. The IDG300 size is $6.0 \times 6.0 \times 1.5 \mathrm{~mm}$, the measurement range is $\pm 500 \mathrm{Deg} / \mathrm{s}$ and the sensitivity is $2.0 \mathrm{mV} / \mathrm{Deg} / \mathrm{s}$. The mixed configuration of two gyroscopes allows the IMU to obtain all the three axis of angular velocity in one planar layer. The output of both gyroscopes is analog signal filtered by a first order low-pass analog filter at cutting frequency $40 \mathrm{~Hz}$.

2) Accelerometer: The 3-axis accelerometer LIS3LV02DL (STMicroelectronics) is a small size $(4.4 \times 7.5 \times 1.0 \mathrm{~mm})$ and high performance MEMs accelerometer. The resolution with a full-scale $\pm 2 \mathrm{G}$ and Bandwidth of $40 \mathrm{~Hz}$ is about $1 \mathrm{mG}$, with noise level of about 0.005 $\mathrm{m} / \mathrm{sec}^{2}$, less than one bit. The accelerometer transmits the data to the microcontroller by using $\mathrm{I}^{2} \mathrm{C}$ communication.

3) Magnetometer: The 3-axis magnetometer HMC5843 (Honeywell), whose size is $4.0 \times 4.0 \times 1.5 \mathrm{~mm}$ is used to measure the local magnetic field. Its full scale is \pm 4 Gauss with a noise level less than two bits. The communication between the magnetometer and microcontroller is performed by $\mathrm{I}^{2} \mathrm{C}$. 
4) Microcontroller: WB-3 contains a 32-bit microcontroller of STM32 (STMicroelectronics) Cortex series for sensor data acquisition, data elaboration and data transmission to PC. In our design, we chose the STM32F103CBT6 with the package LQFP48 (7.0x7.0x1.4 mm). The STM32F103CBT6 incorporates a high performance ARM® Cortex ${ }^{\text {TM}}-\mathrm{M} 3$ 32-bit RISC core operating up to $72 \mathrm{MHz}$, and high-speed embedded memories (Flash memory 128 Kbytes).

The characteristics of the WB-3 sensors are all summarized in Table I.

The size, the sensor bandwidth and range of WB-3 are compatible with the normal requirements of the human motion analysis both for the upper limbs [33],[34], and the lower limbs [35],[36] . WB-3 IMU has already been successfully applied for the analysis of the human body movements in medical application[37-40].

Table I Main characteristics of the WB-3 sensors.

\begin{tabular}{ccccc}
\hline \hline & LIS3LV02DL & IDG300 & LISY300AL & HMC5843 \\
\hline Category & Accelerometer & Gyroscope & Gyroscope & Magnetometer \\
\hline Axis & 3-Axis & 2-Axis & 1-Axis & 3 -Axis \\
\hline Range & $\pm 2 \mathrm{G} / \pm 6 \mathrm{G}$ & $\pm 500 \mathrm{Deg} / \mathrm{s}$ & $\pm 300 \mathrm{Deg} / \mathrm{s}$ & $\pm 4 \mathrm{Gauss}$ \\
\hline Resolution & $12 \mathrm{bit}$ & $12 \mathrm{bit}$ & $12 \mathrm{bit}$ & $12 \mathrm{bit}$ \\
\hline Bandwidth & $40 \mathrm{~Hz}$ & $140 \mathrm{~Hz}$ & $88 \mathrm{~Hz}$ & $40 \mathrm{~Hz}$ \\
\hline Linearity & $\pm 2 \%$ & $<1 \%$ & $\pm 0.8 \%$ & $\pm 0.1 \%$ \\
\hline Noise Level & $<1 \mathrm{bit}$ & $<2 \mathrm{bit}$ & $<2 \mathrm{bit}$ & $<2 \mathrm{bit}$
\end{tabular}

\subsubsection{Attitude estimation algorithm}

In the IMUs, the main problem is that the orientation is computed by the integration of the gyro signals including any superimposed sensor drift and noise. Additional sensors such as accelerometer and magnetometer can bound the orientation drift errors resulting from gyroscope output errors, by using an Extended Kalman Filter (EKF). For example, the earth's gravitational field vectors in the body frame can be obtained with their known representation in the absolute reference frame [41]. In the WB-3 algorithm, a state augmentation technique is applied in the process model, and the state vector is composed by orientation and gyro bias [42]. Quaternions are used to represent space orientation to improve computational efficiency and avoid singularities [43]. The EKFcomputed quaternion can be translated into Roll, Pitch and Yaw angles through transformation equations (Section 3.1). 
The continuous-time, non-linear system equations for the IMU are:

$$
\begin{aligned}
& \dot{x}=f(x, \omega)+w \\
& y=h(x)+v
\end{aligned}
$$

where $x=\left[{ }_{G L}^{W B} q_{W B}, b_{\omega}\right]$ represents the state of the system composed by: the quaternion ${ }_{G L}^{W B} q_{W B}={ }_{G L}^{W B}\left\lfloor q_{W B_{0}}, q_{W B_{1}}, q_{W B_{2}}, q_{W B_{3}}\right\rfloor$ measured by the WB-3 for the rotation from the global reference frame $G L$, identified by the magnetic north and the gravity vector, to the sensor frame of the WB-3 (WB) [32]; and the rate gyro bias $b_{\omega}=\left[b_{\omega_{x}}, b_{\omega_{y}}, b_{\omega_{z}}\right] . \omega=\left[\omega_{x}, \omega_{y}, \omega_{z}\right]$ is the angular rates vector; $y=[a, m]$ is the measurement vector composed by the acceleration measurements $a=\left[a_{x}, a_{y}, a_{z}\right]$ and the magnetometer measurements $m=\left[m_{x}, m_{y}, m_{z}\right]$.

Finally, $w$ and $v$ are the additive state noise and measurement noise respectively. The state and measurement noise are Gaussian and white noise sources with covariance matrix $Q$ and $R$ respectively.

In a strap-down inertial navigation system, the rigid body angular motion is described by the following differential equation:

$$
{ }_{G L}^{W B} \dot{q}_{W B}=\frac{1}{2}\left[\begin{array}{cc}
0 & -\omega^{T} \\
\omega & -[\omega \times
\end{array}\right]{ }_{G L}^{W B} q_{W B}
$$

where $[\omega \times]$ is the cross product of the angular velocity

$$
[\omega \times]=\left[\begin{array}{ccc}
0 & -\omega_{z} & \omega_{y} \\
\omega_{z} & 0 & -\omega_{x} \\
-\omega_{y} & \omega_{x} & 0
\end{array}\right]
$$

with the assumption that $\|q\|=1$

Using the sensor model described in [44], in quasi static condition, the acceleration acting on the body is negligible compared to the gravity acceleration as described by:

$$
\left\{\begin{array}{l}
\omega=G_{g} \omega_{r}+b_{\omega}+v_{g} \\
a=G_{a}\left({ }_{G L}^{W B} C \cdot g\right)+v_{a} \\
m=G_{m}\left({ }_{G L}^{W B} C \cdot H\right)+v_{m}
\end{array}\right.
$$

where $G_{g}, G_{a}$, and $G_{m}$ are respectively gyroscope, accelerometer, and magnetometer gain, supposed constant; $v_{g}, v_{a}$, and $v_{m}$ are respectively gyroscope, accelerometer and magnetometer noise; $\omega$ is vector of the gyroscope raw data; ${ }_{G L}^{W B} C$ is the direction cosine matrix in terms of orientation quaternion, that 
represents the coordinate transformation from the absolute coordinate systems $(G L)$, identified by the magnetic north and the gravity vector, to the sensor frame of the WB-3 (WB):

$$
{ }_{G L}^{W B} C=\left[\begin{array}{ccc}
q_{W B_{0}}^{2}-q_{W B_{1}}^{2}-q_{W B_{2}}^{2}+q_{W B_{3}}^{2} & 2\left(q_{W B_{0}} q_{W B_{1}}+q_{W B_{2}} q_{W B_{3}}\right) & 2\left(q_{W B_{0}} q_{W B_{2}}-q_{W B_{1}} q_{W B_{3}}\right) \\
2\left(q_{W B_{0}} q_{W B_{1}}-q_{W B_{2}} q_{W B_{3}}\right) & -q_{W B_{0}}^{2}+q_{W B_{1}}^{2}-q_{W B_{2}}^{2}+q_{W B_{3}}^{2} & 2\left(q_{W B_{1}} q_{W B_{2}}+q_{W B_{0}} q_{W B_{3}}\right) \\
2\left(q_{W B_{0}} q_{W B_{2}}+q_{W B_{1}} q_{W B_{3}}\right) & 2\left(q_{W B_{1}} q_{W B_{2}}-q_{W B_{2}} q_{W B_{3}}\right) & -q_{W B_{0}}^{2}-q_{W B_{1}}^{2}+q_{W B_{2}}^{2}+q_{W B_{3}}^{2}
\end{array}\right]
$$

$g=[0,0,1]^{T}$ is the gravity vector (measured in G), $H=\left[H_{x}, 0, H_{z}\right]^{T}$ is the local magnetic field in the inertial frame. The local magnetic field has the y component equal to zero because we assume the magnetic north direction orthogonal to the $y$ axis of the absolute coordinate system. The gravity vector has the $\mathrm{x}$ and $\mathrm{y}$ components equal to zero because we assume that the absolute coordinate system has the $\mathrm{z}$ axis aligned with the gravity vector (in the same direction).

The non-linear functions and $h(x)$ can be explained as:

$$
h(x)=\left[\begin{array}{l}
W B \\
G L \\
{ }_{W B} \\
{ }_{G L}
\end{array}\right.
$$

The estimation step of the EKF uses the angular rate to make a prediction of the state. Furthermore, the acceleration data is used to correct Roll and Pitch angles in the "Roll-Pitch Update" block as showed in the Figure 5. The correction of the yaw angle "Yaw Update" can be performed using the magnetometer (Magnetic North). Magnetic interference is also a major concern when using magnetometers in environments containing changing or distorted magnetic fields, but adaptive compensation for external magnetic effects is not performed in this work. The problems and limitations of the magnetic correction in the EKF are under further investigation from the authors and several other researchers [45-48].

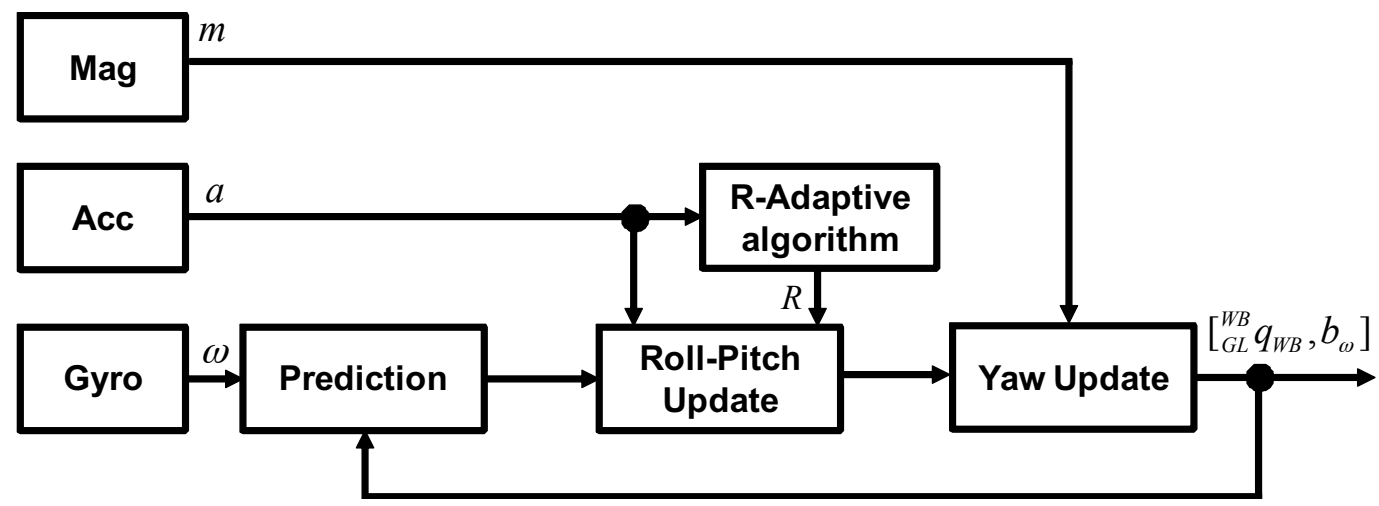

Figure 5: Block diagram of the EKF algorithm implemented for WB-3. 


\subsubsection{R-Adaptive algorithm}

The main limitation of the algorithm described in the previous section is related to the "quasi-static condition", in which the linear accelerations are negligible compared to the gravity acceleration. In the case of human motion tracking (i.e. during walking or running), often this condition is not verified. For example, if the IMU is positioned on the foot, during the swing phase of human walking a linear acceleration from $2 \mathrm{G}$ to $5 \mathrm{G}$ is quite common [49].

To overcome the problems related to the correction of the attitude using the data from the accelerometer, several techniques have been previously used [50], [51]. Some other techniques monitor the angular rate and the module of acceleration to determine quasi-static conditions. If a quasi-static condition is detected, the Update phase of the EKF is performed, otherwise it is just skipped. These techniques solve quite efficiently the drawback of the EKF, however the threshold that discriminates between the dynamic and static conditions must be chosen carefully. Furthermore, it is difficult to set the coefficients of the measurement covariance matrix (R). Not only the measurement noise, but also the error due to the linear accelerations should be considered for a correct evaluation. To overcome these problems, the R-adaptive algorithm has been implemented by the authors [30].

$R$ is assumed to be in this form:

$$
R=\left[\begin{array}{ccc}
\sigma_{x}^{2} & 0 & 0 \\
0 & \sigma_{y}^{2} & 0 \\
0 & 0 & \sigma_{z}^{2}
\end{array}\right]
$$

where $\sigma_{x}^{2}, \sigma_{y}^{2}$, and $\sigma_{z}^{2}$ are the standard deviations of the measurements for the $\mathrm{x}, \mathrm{y}$ and $\mathrm{z}$ axis of the acceleration sensors respectively.

Considering the sensors axis with the same noise characteristics $\left(\sigma_{x}^{2}=\sigma_{y}^{2}=\sigma_{z}^{2}=\sigma^{2}\right), \sigma^{2}$ can be estimated dynamically in a temporal window with the following formula:

$$
\sigma_{k}^{2}=\frac{1}{N+1} \sum_{i=k-N}^{k}\left(\left\|a_{i}\right\|-1\right)^{2}
$$

where $N$ is the number of samples of the temporal window; $\sigma_{k}^{2}$ is the estimated variance at step $k ;\left\|a_{i}\right\|$ is the module of the acceleration measured by the accelerometer (in $\mathrm{G})$ at the step $i$. 
The equation (16) is a good approximation of the variance for the following reasons. In static conditions, for $N \rightarrow \infty$, the calculated variance is exact. Furthermore, in dynamic conditions, it provides a variance value, which measures the dispersion of the acceleration module in respect to the gravity. The authors heuristically fixed the temporal windows at $N=30$ samples (@200Hz) and $N=15$ samples (@100Hz) because these values provides a good approximation of the variance in static conditions and give a fast response on the dynamic conditions in the case of human movements.

\subsection{InertiaCube3 ${ }^{\mathrm{TM}}$}

The InertiaCube $3^{\mathrm{TM}}$ is an inertial measurement system that provides full $360^{\circ}$ source-less tracking in all axes. The InertiaCube $3^{\mathrm{TM}}$ integrates nine discrete, miniature-sensing elements with proprietary advanced Kalman filtering algorithm. The sensor can be connected to a host PC by using USB interface.

Heading calibration software compensates for static magnetic field distortions when the InertiaCube $3^{\text {TM }}$ is deployed in adverse environments.

The InertiaCube3 ${ }^{\mathrm{TM}}$ has size $26.2 \times 39.2 \times 14.8 \mathrm{~mm}$ and weight $17.0 \mathrm{~g}$ (with housing). On the Table II, the main characteristics of the InertiaCube ${ }^{\mathrm{TM}}$ are showed.

Table II Main characteristics of the InertiaCube $3^{\mathrm{TM}}$.

\begin{tabular}{|c|c|}
\hline $\begin{array}{l}\text { Degrees of Freedom } \\
\text { (angle representation) }\end{array}$ & 3 (Yaw, Pitch and Roll) \\
\hline Maximum Angular Rate & $1200 \% / \mathrm{s}$ \\
\hline Minimum Angular Rate & $0 \% / \mathrm{s}$ \\
\hline RMS Accuracy* & $1^{\circ}$ in yaw, $0.25^{\circ}$ in pitch $\&$ roll at $25^{\circ} \mathrm{C}$ \\
\hline RMS Angular Resolution* & $0.03^{\circ}$ \\
\hline InterSense USB Update Rate & $180 \mathrm{~Hz}$ \\
\hline $\begin{array}{c}\text { USB Interface Minimum } \\
\text { Latency }\end{array}$ & $2 \mathrm{~ms}$ for USB direct (Host \& OS dependent) \\
\hline Operating Temperature Range & $0^{\circ}$ to $70^{\circ} \mathrm{C}$ \\
\hline
\end{tabular}

The WB-3 and Vicon system have a selectable sample rate up to $200 \mathrm{~Hz}$, which is enough for the majority of the applications in the human body movement analysis [51]. The commercial IMU (InertiaCube ${ }^{\mathrm{TM}}$ ) samples internally the sensors at 180 $\mathrm{Hz}$ but the sample rate of the attitude angle output by the sensor acquired with a HP Pavilion dv6600 (Intel Core 2 Duo T7250 - RAM 2GB) on the OS Windows7 was quite variable with a main sample frequency of about $40 \mathrm{~Hz}$ (every $25 \mathrm{~ms}$ ). As shown in Figure 6, the sample rate for the WB-3 is very stable, $100 \%$ of the 
samples were transmitted with a sample time of $5 \mathrm{~ms}(@ 200 \mathrm{~Hz})$. InertiaCube $3^{\mathrm{TM}}$ instead transmits only $77 \%$ of the data every $25 \mathrm{~ms}$, and a significant percentage of the data, more than $12 \%$, were sent with a sampling rate over $80 \mathrm{~ms}$. The data of the InertiaCube $3^{\mathrm{TM}}$ have been re-sampled in post-elaboration at the same frequency of the Vicon for an effective comparison with the other systems.

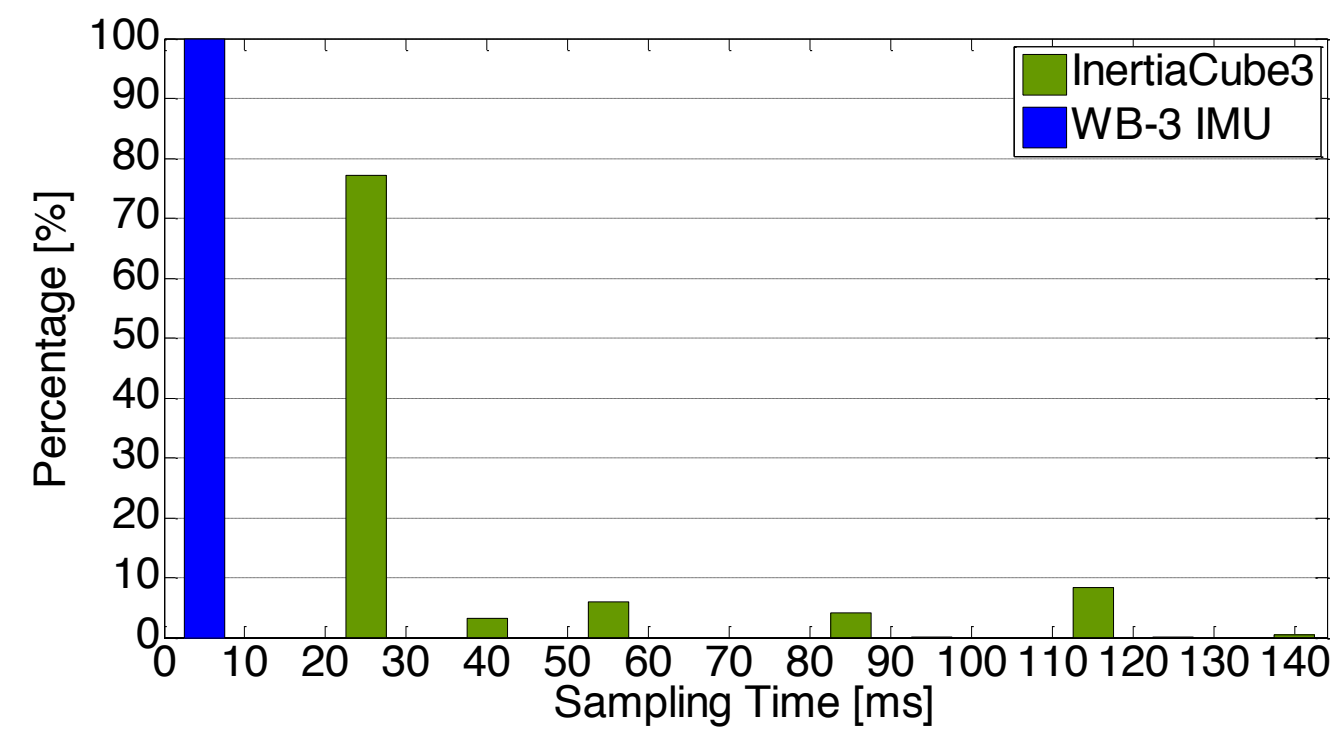

Figure 6: Histogram of the Sample Time WB-3 and InertiaCube3 ${ }^{\mathrm{TM}}$.

The InertiaCube $3^{\mathrm{TM}}$ provides the attitude data using an ZYX Euler representation also known as Roll, Pitch, Yaw (RPY).

The conversion equations from Roll $\left(\theta_{I C}\right)$, Pitch $\left(\phi_{I C}\right)$, Yaw $\left(\psi_{I C}\right)$ to the correspondent quaternion ${ }_{G L}^{I C} q_{I C}={ }_{G L}^{I C}\left[q_{I C_{0}}, q_{I C_{1}}, q_{I C_{2}}, q_{I C_{3}}\right]$ were used to make the angle representation homogeneous for the algorithm of data synchronization and frame alignment. The equations used for the conversion are:

$$
\begin{aligned}
& q_{I C_{0}}=\cos \left(\frac{\phi_{I C}}{2}\right) \cos \left(\frac{\theta_{I C}}{2}\right) \cos \left(\frac{\psi_{I C}}{2}\right)+\sin \left(\frac{\phi_{I C}}{2}\right) \sin \left(\frac{\theta_{I C}}{2}\right) \sin \left(\frac{\psi_{I C}}{2}\right) \\
& q_{I C_{1}}=\sin \left(\frac{\phi_{I C}}{2}\right) \cos \left(\frac{\theta_{I C}}{2}\right) \cos \left(\frac{\psi_{I C}}{2}\right)-\cos \left(\frac{\phi_{I C}}{2}\right) \sin \left(\frac{\theta_{I C}}{2}\right) \sin \left(\frac{\psi_{I C}}{2}\right) \\
& q_{I C_{2}}=\cos \left(\frac{\phi_{I C}}{2}\right) \sin \left(\frac{\theta_{I C}}{2}\right) \cos \left(\frac{\psi_{I C}}{2}\right)+\sin \left(\frac{\phi_{I C}}{2}\right) \cos \left(\frac{\theta_{I C}}{2}\right) \sin \left(\frac{\psi_{I C}}{2}\right) \\
& q_{I C_{3}}=\cos \left(\frac{\phi_{I C}}{2}\right) \cos \left(\frac{\theta_{I C}}{2}\right) \sin \left(\frac{\psi_{I C}}{2}\right)-\sin \left(\frac{\phi_{I C}}{2}\right) \sin \left(\frac{\theta_{I C}}{2}\right) \cos \left(\frac{\psi_{I C}}{2}\right)
\end{aligned}
$$




\section{Methodology}

\subsection{The methodology for the performance comparison}

The main purpose of the proposed methodology is to evaluate the performance of several attitude sensors comparing them with the attitude provided by the Vicon motion system. The methodology is quite general and can be applied for any kind of sensor that gives information about the attitude of an object on the space. In this paper, we focused on the performance evaluation of the WB-3 IMU and the commercial IMU, InertiaCube $3^{\mathrm{TM}}$. A preprocessing stage is needed for each sensor, as described in the previous sections, to obtain the attitude data in a quaternion representation. The data of the sensors are re-sampled at the same sample rate of the Vicon considered as ground truth. The data processing flow diagram for the performance comparison is showed in Figure 7. All the errors are expressed in terms of Euler angles because it is visually more intuitive to compare the error in terms of angle rather than quaternion.

If we indicate with $q=\left[q_{0}, q_{1}, q_{2}, q_{3}\right]$ the generic quaternion, the conversion equations used to obtain Roll $(\theta)$, Pitch $(\varphi)$, Yaw $(\psi)$ are the following:

$$
\begin{aligned}
& \phi=a \tan 2\left(2\left(q_{1} q_{2}+q_{3} q_{0}\right),-1+2\left(q_{2}^{2}+q_{3}^{2}\right)\right) \\
& \theta=\arccos \left(-2\left(q_{1} q_{3}-q_{0} q_{2}\right)\right) \\
& \psi=a \tan 2\left(2\left(q_{0} q_{1}+q_{2} q_{3}\right),-1+2\left(q_{0}^{2}+q_{3}^{2}\right)\right.
\end{aligned}
$$

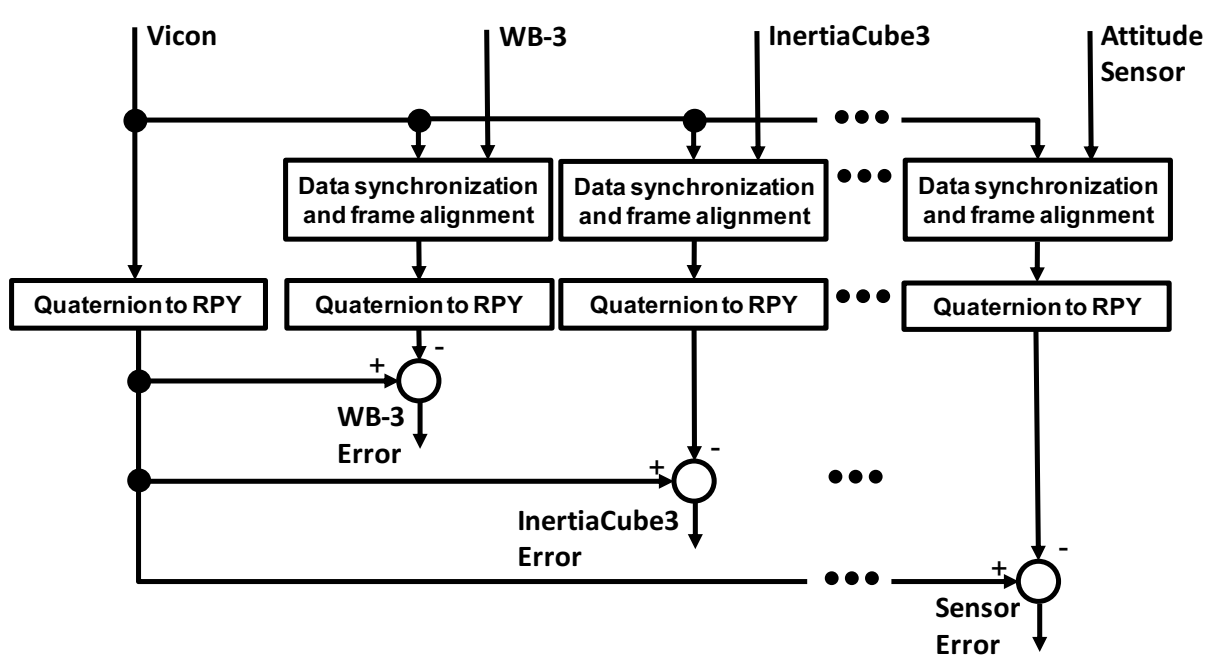

Figure 7: Data processing flow diagram for the performance comparison among attitude sensors (WB-3, InertiaCube ${ }^{\mathrm{TM}}$, and so on). The reference truth is the Vicon systems. 


\subsection{Data Synchronization and frame alignment}

The fundamental stage for the coherent performance comparison among the different systems is the data synchronization and frame alignment. The Vicon and the IMUs data cannot be compared unless they are perfectly synchronized. There are not synchronization issues between sensors that are captured on the same computer if it is possible to evaluate embedded timestamps for the respective hardware [52]. Unfortunately, more often the sensors allow only a software timestamp that recognize only when the data is received, not when the data is acquired, introducing errors due to a random network delay. The IMUs and Vicon data cannot be synchronized by direct comparison for two main reasons. Firstly, different computers collected the data from the sensors, and the reference systems are different, IMUs measure the attitude in the global frame $(G L)$ while the Vicon measure the attitudes with respect its global frame (VI). A genetic algorithm coupled with Dynamic Time Warping (DTW), as shown in Figure 8, was implemented overcome the misalignment issues among the reference systems (Vicon and IMUs) and the synchronization problem.

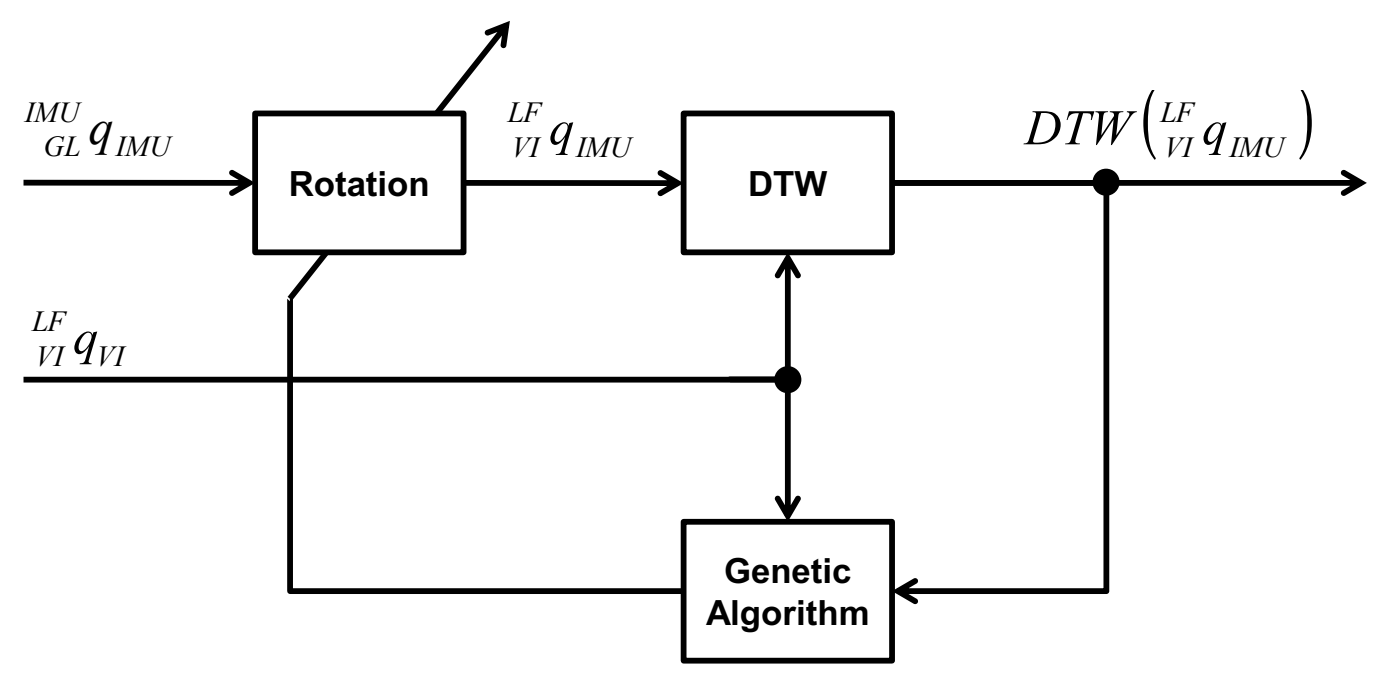

Figure 8: Data synchronization and frame alignment block diagram.

The inputs of the algorithm are the angles expressed in quaternion ${ }_{V I}^{L F} q_{V I}$ and ${ }_{G L}^{I M U} q_{I M U}$, where $I M U$ is either $W B(W B-3)$ or $I C\left(\right.$ InertiaCube $\left.3^{\mathrm{TM}}\right)$.

The output of the algorithm is $D T W\left({ }_{V I}^{L F} q_{I M U}\right)$ which is the attitude of the LFrame measured by the IMU in the reference frame of the Vicon synchronized using the DTW algorithm. The rotation matrix is adapted by the Genetic Algorithm to minimize the error between the $D T W\left({ }_{V I}^{L F} q_{I M U}\right)$ and the ${ }_{V I}^{L F} q_{V I}$. The 
rotation matrix obtained by the genetic algorithm is then used to obtain ${ }_{V I}^{L F} q_{I M U}$, the attitude of the L-Frame measured by the IMU in the reference frame of the Vicon.

\subsection{Dynamic Time Warping}

The DTW algorithm is a powerful algorithm that allows the comparison of time series for a proper synchronization.

Normally, the Euclidean distance is an efficient distance measurement that can be used to determine similarity between synchronized time series. Unfortunately, if two time series are identical, but one is shifted slightly along the time axis due to a no-perfect synchronization or variation of the sampling time, then Euclidean distance may consider them very different from each other. In Figure 9, the effect of the synchronization problem is clearly visible. The signal $\cos (2 \pi t)$ is compared with the unsynchronized signal. We added a fix delay of $0.1 \mathrm{~s}$ and a random delay signal uniformly distributed in the range [-0.01s $0.01 \mathrm{~s}]$. Even the two signals are originally the same, the error calculated as simple difference is very large (with peaks of \pm 0.6 ) especially when the two signals change rapidly.

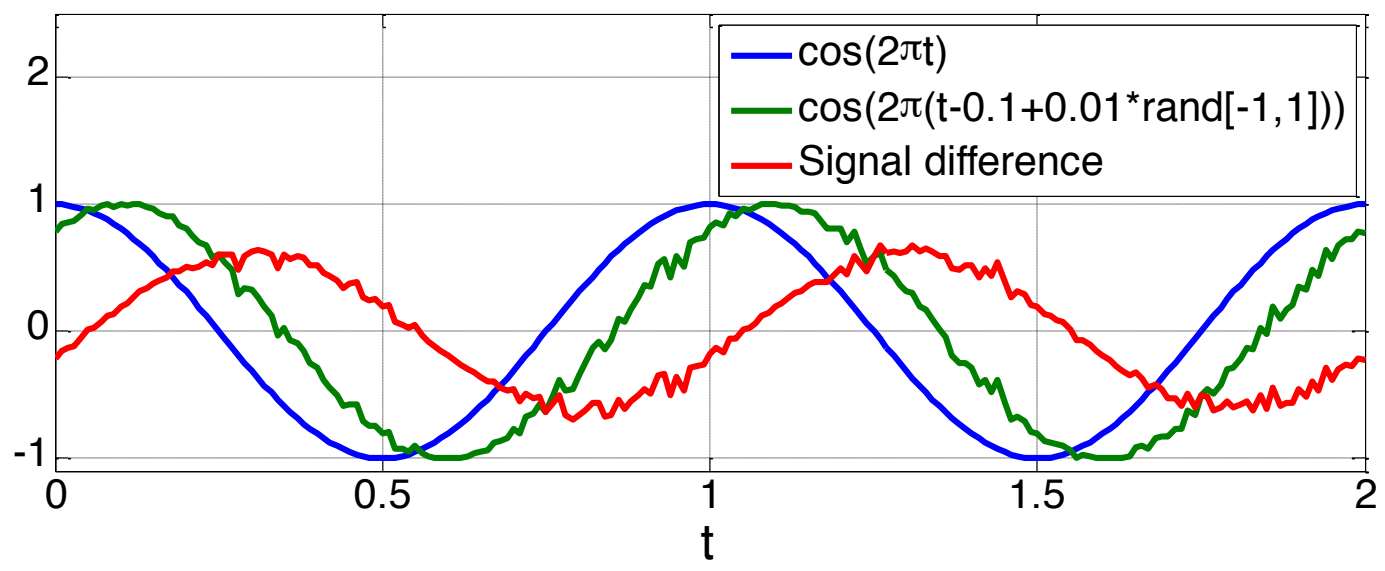

Figure 9: Effect of the synchronization issues for the comparison of signals.

To overcome this kind of problem and give intuitive distance measurements between time series, the Dynamic Time Warping (DTW) algorithm has been introduced in [53]. DTW algorithm scales the time dimension reducing the problems of both global (fix delay) and local shifts in the time (random changes on the sample frequency). DTW has earned popularity to be extremely efficient to find similarities among time series. Given two time series $S^{a}=\left(s^{a}{ }_{1}, s^{a}{ }_{2}, \ldots, s_{U_{a}}\right)$ with $U_{a} \in \mathcal{K}$ and $S^{b}=\left(s_{1}^{b}, s^{b}{ }_{2}, \ldots, s^{b}\right) U_{b} \in \mathcal{K}$, the DTW algorithm minimizes 
the effects of shifting and time distortion allowing "elastic" transformation of time series to detect similar shapes with different phases. If the time series are taking values from a feature space $\Phi$, then a local distance measure $d$ is defined to compare the two different sequences $S^{a}, S^{b} \in \Theta$

$$
d: \Phi \times \Phi \rightarrow \Re \geq 0
$$

$d$ is called "distance" or "cost" function, and it has small values when the sequences are similar and large when they are different.

The DTW builds a distance matrix $\Psi \in \mathfrak{R}^{U_{a} \times U_{b}}$, named also local cost matrix that represents all the pair wise distances between $S^{a}$ and $S^{b}$.

$$
\Psi \in \mathfrak{R}^{U_{a} \times U_{b}}: \alpha_{i, j}=\left\|s^{a}{ }_{i}-s^{a}{ }_{j}\right\|
$$

with $i \in\left[1: U_{a}\right], j \in\left[1: U_{b}\right]$.

Once the matrix is built, the DTW finds the alignment path, or warping path, that defines the correspondences $s^{a}{ }_{i}$ to $s^{a}{ }_{j}$. The alignment path is a sequence of points $P=\left(p_{1}, p_{2}, \ldots p_{k}\right) \quad$ with $\quad p_{l}=\left(p_{i}, p_{j}\right) \in\left[1: U_{a}\right] \times\left[1: U_{b}\right]$ for $l \in[1: k]$ which satisfies the following criteria:

1) Boundary condition: $p_{1}=(1,1)$ and $p_{k}=\left(U_{a}, U_{b}\right)$

2) Monotonicity: $s^{a}{ }_{1} \leq S^{a}{ }_{2} \leq \ldots \leq s^{a}{ }_{k}$ and $s^{b}{ }_{1} \leq S^{b}{ }_{2} \leq \ldots \leq S^{b}{ }_{k}$

3) Step size condition: this criterion limits the warping path from long jumps (shifts) while aligning sequences.

To find the optimal path, the algorithm should calculate all the possible paths and select only the best one. This method is computational heavy because it needs a complete search all over the possible paths.

The DTW employs the dynamic programming to keep the complexity to only $\mathrm{O}\left(\mathrm{U}_{\mathrm{a}} \mathrm{U}_{\mathrm{b}}\right)$. The dynamic programming is based on the accumulated cost matrix and the optimal path can be found by backtracking from $p_{k}=\left(U_{a}, U_{b}\right)$ to $p_{1}=(1,1)$ following the greedy strategy [54].

\subsection{Genetic algorithm}

The Genetic algorithm used a population of two rotations. The first rotation represents the transformation between the Global reference system (GL) and the Vicon reference system (VI). Furthermore, a second rotation is necessary to align the IMU reference frame (IMU) and the L-frame reference frame (LF). 
A couple of unit quaternion can represent the two rotations sequences as follows:

$$
\left({ }_{G L}^{V I} q,{ }_{I M U}^{L F} q\right)
$$

Because of the quaternion representation, we need to search in a domain of only eight parameters for the optimal succession of rotations. The fitness function used to find the optimal solution is the follow:

$$
\text { fitness }=R M S\left(\|\|_{G L}^{L F} q_{V I}-D T W\left({ }_{G L}^{L F} q_{I M U}\right) \|\right)
$$

The fitness function is the root mean square (RMS) of the norm of the quaternion error between the attitude estimation of the Vicon and the warped data of the IMU. It is important to notice the following equations that indicate the relation with the misalignment of the reference system:

$$
\begin{aligned}
& { }_{G L}^{L F} q_{I M U}={ }_{G L}^{I M U} q_{I M U} \quad{ }_{I M U}^{L F} q \\
& { }_{G L}^{L F} q_{V I}={ }_{G L}^{V I} q_{V I}{ }_{V I}^{L F} q
\end{aligned}
$$

In the present work, we used a population of 500 couples of quaternions performing 200 iterations of generation. Half of the population is chosen among the best fitting individuals; the other half is obtained breeding new individuals through crossover.

\section{Experimental Validation}

We validated the comparison methodology performing two types of experiments at different sampling rate, $100 \mathrm{~Hz}$ and $200 \mathrm{~Hz}$.

For each sample rate, we rotated the L-Frame at different speeds. It is important to observe that the sensors are not placed on the rotation center of the L-Frame. Therefore, the sensors are also subjected to linear accelerations that depend on the rotation speed of the L-frame.

We validated the proposed methodology measuring the performance of the IMUs using the following four experiments:

- Acquisition of the Vicon data at $100 \mathrm{~Hz}$

○ Experiment A: 45 rotations around the X1 (Roll) at different speeds, for a total of 100 seconds of continuous acquisition;

- Experiment B: free rotations for a total of 50 seconds of continuous acquisitions at $100 \mathrm{~Hz}$.

- Acquisition of the Vicon data at $200 \mathrm{~Hz}$ 
○ Experiment C: 18 rotations around X1 at different speeds, for a total of 40 seconds of continuous acquisition;

○ Experiment D: 17 rotations around the Y1 at different speeds, for a total of 40 seconds of continuous acquisition;

During these movements, the orientation data are computed by the WB-3, InertiaCube $3^{\mathrm{TM}}$, and the Vicon. All the data are logged and off-line compared.

The resulting average RMS errors for the Roll and Pitch are presented in Tables III-VI. Both, WB-3 and InertiaCube $3^{\mathrm{TM}}$ sensors measure the attitude relatively well. The maximum RMS error for the InertiaCube ${ }^{\mathrm{TM}}$ was respectively $5.92 \mathrm{Deg}$ for the Pitch angle, obtained in the experiment B conducted at $100 \mathrm{~Hz}$, and 6.57 Deg for the Roll angle, obtained in the experiment C conducted at $200 \mathrm{~Hz}$. Therefore, we can say that, in the case of the InertiaCube $3^{\mathrm{TM}}$, the angle accuracy of the InertiaCube ${ }^{\mathrm{TM}}$ of the system can be reasonable considered about 6.6 Deg. These results are not in contrast to the specifications provided by the sensor maker because the values of accuracy provided in Table II are related to static conditions while we performed experiments in dynamic conditions.

The maximum RMS error for the WB-3 was respectively 2.69 Deg for the Pitch angle, obtained in the experiment D conducted at $200 \mathrm{~Hz}$, and $3.32 \mathrm{Deg}$ for the Yaw angle obtained in the experiment B. In this case, we could reasonably claim that the angle accuracy of the WB-3 is about 3.4 Deg.

InertiaCube $3^{\text {TM }}$ had better performance than WB-3 for the Roll and Pitch angles estimation in the Experiment D. Although WB-3 IMU had worse performance than InertiaCube $3^{\mathrm{TM}}$ in the specific experiment, it still had reasonable dynamic performance, consistently with the other trials.

The resulting angles for the experiment B related to the free rotation (Roll and Pitch angles) are presented in Figure 10. The WB-3 angles fit better the Vicon angles. Furthermore, the WB-3 angles are smoother than the InertiaCube $3^{\mathrm{TM}}$ that presents some glitches that might be due to the linear acceleration during the movement.

Figure 11 shows the reconstructed Roll angles for the experiment $\mathrm{C}$. The figures clearly show better dynamic performance of WB-3. InertiaCube $3^{\text {TM }}$ had bigger errors compare to the WB-3 at the peaks of the signal, which were the turning moments of the rotation movements. At these turning points, there were strong changes of angular velocity, which will result in big variance of centripetal 
acceleration. This effect might affect the performance of "Roll-Pitch Update" in the EKF. WB-3 could adapt to this condition better by taking advantage of the Radaptive algorithm.

It is also important to notice that, the attitude reconstruction algorithm of InertiaCube $3^{\text {TM }}$ does not work if the pitch angle is around 90 Deg. because of singularity problems due to the Euler angle representations. The WB-3 algorithm is not affected by this problem because of the quaternion-based representation of the angles.

Table III: Performance evaluation at $100 \mathrm{~Hz}$ (Experiment $\mathrm{A})$.

\begin{tabular}{cccc}
\hline \hline & $\begin{array}{c}\text { RMS Roll Error } \\
{[\text { Deg] }}\end{array}$ & $\begin{array}{c}\text { RMS Pitch Error } \\
{[\text { Deg }]}\end{array}$ & $\begin{array}{c}\text { RMS Yaw Error } \\
{[\text { Deg }]}\end{array}$ \\
\hline WB-3 IMU & 1.23 & 0.9 & 2.80 \\
\hline InertiaCube3 & 2.25 & 1.07 & 2.52 \\
\hline
\end{tabular}

Table IV: Performance evaluation at $100 \mathrm{~Hz}$ (Experiment B).

\begin{tabular}{cccc}
\hline \hline & $\begin{array}{c}\text { RMS Roll Error } \\
{[\mathrm{Deg}]}\end{array}$ & $\begin{array}{c}\text { RMS Pitch Error } \\
{[\mathrm{Deg}]}\end{array}$ & $\begin{array}{c}\text { RMS Yaw Error } \\
{[\mathrm{Deg}]}\end{array}$ \\
\hline WB-3 IMU & 1.06 & 2.54 & 3.32 \\
\hline InertiaCube3 & 2.07 & 5.92 & 2.90 \\
\hline
\end{tabular}

Table V: Performance evaluation at $200 \mathrm{~Hz}$ (Experiment C).

\begin{tabular}{cccc}
\hline \hline & $\begin{array}{c}\text { RMS Roll Error } \\
{[\mathrm{Deg}]}\end{array}$ & $\begin{array}{c}\text { RMS Pitch Error } \\
{[\mathrm{Deg}]}\end{array}$ & $\begin{array}{c}\text { RMS Yaw Error } \\
{[\mathrm{Deg}]}\end{array}$ \\
\hline WB-3 IMU & 2.22 & 0.63 & 1.80 \\
\hline InertiaCube3 $^{\mathrm{TM}}$ & 6.57 & 2.94 & 4.52 \\
\hline
\end{tabular}

Table VI: Performance evaluation at $200 \mathrm{~Hz}$ (Experiment D).

\begin{tabular}{cccc}
\hline \hline & $\begin{array}{c}\text { RMS Roll Error } \\
{[\text { Deg }]}\end{array}$ & $\begin{array}{c}\text { RMS Pitch Error } \\
{[\text { Deg }]}\end{array}$ & $\begin{array}{c}\text { RMS Yaw Error } \\
{[\text { Deg }]}\end{array}$ \\
\hline WB-3 IMU & 1.88 & 2.69 & 1.32 \\
\hline InertiaCube3 & 1.06 & 1.53 & 1.40 \\
\hline
\end{tabular}



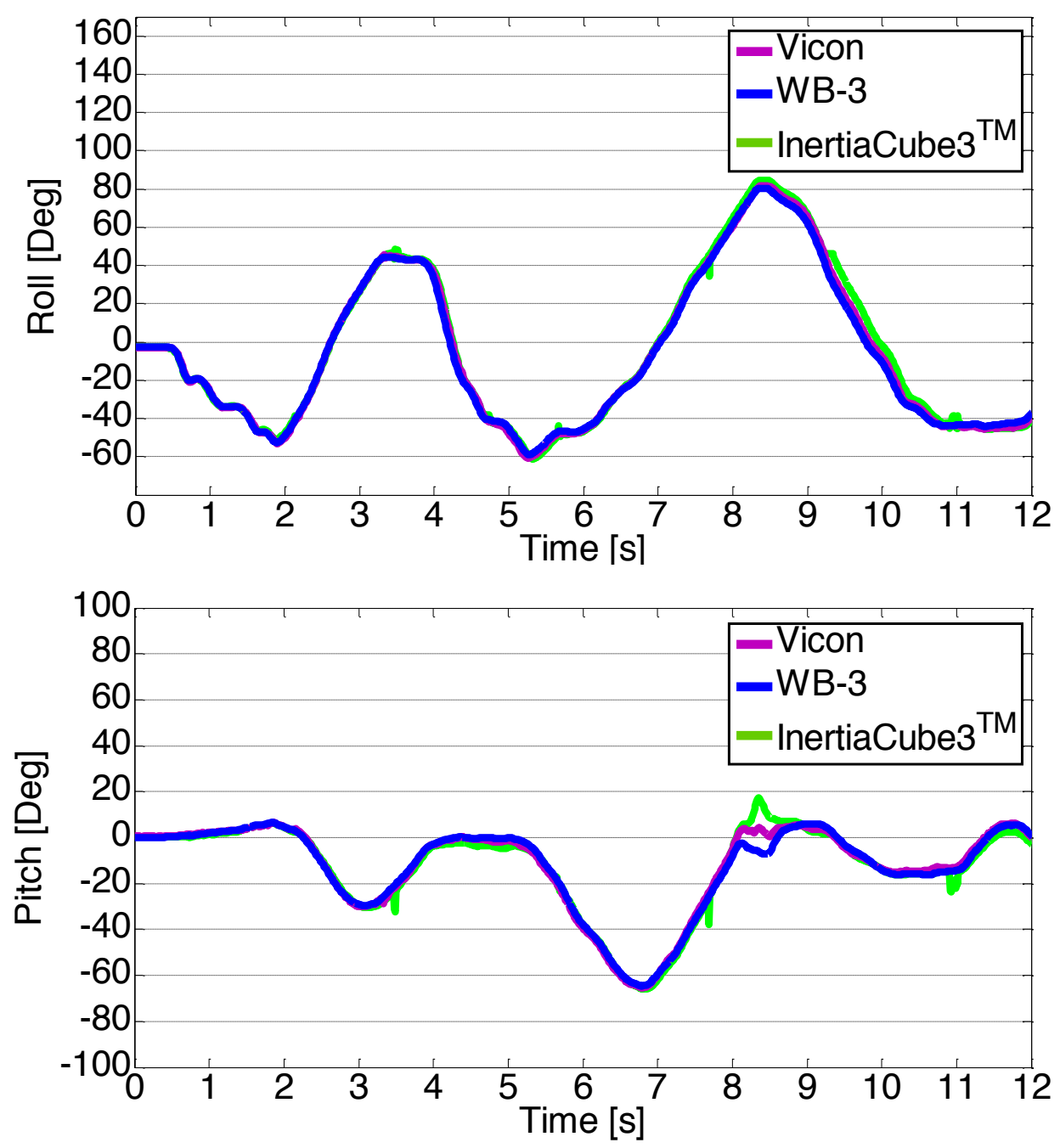

Figure 10: Experiment $B$ - WB-3 and InertiaCube3 ${ }^{\text {TM }}$ Roll angle (TOP) and Pitch angle (BOTTOM) compared with Vicon in the range 0 - 12 s during free rotations. 


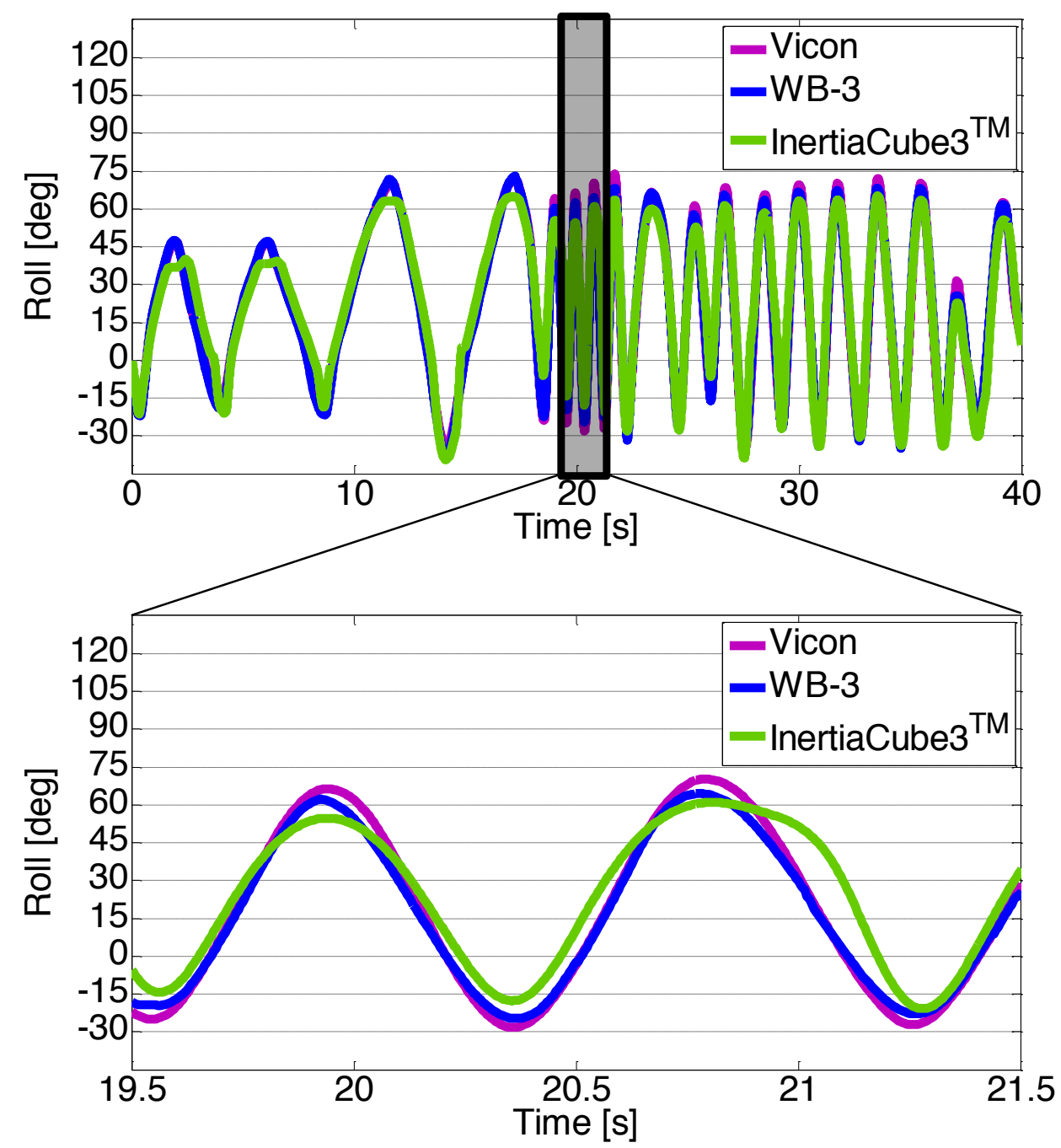

Figure 11: Experiment $C$ - WB-3 and InertiaCube3 ${ }^{\mathrm{TM}}$ roll angle compared with Vicon for the full experiment (TOP) and a zoom of the signal in the range 19.5 21.5 s (BOTTOM).

\section{$5 \quad$ Conclusion and Future Work}

In this paper, we presented a methodology for a reliable comparison among Inertial Measurement Units, or other devices for the attitude measurement, in a Vicon environment as reference truth.

We solved the major issues of data synchronization and alignments of the reference frames by using an original algorithm based on the coupling of a DTW and genetic algorithm.

The proposed methodology has been successfully applied for the performance comparison between the WB-3 and the InertiaCube $3^{\mathrm{TM}}$. In particular, the 
experimental results showed that the performances of WB-3 are superior to the InertiaCube ${ }^{\mathrm{TM}}$. Furthermore, WB-3 provides a good attitude reconstruction in respect to the Vicon system that is actually the basic tool for the body movement analysis in clinic environment.

Another, important application of the proposed synchronization method is on the data synchronization between the sensors embedded on mobile robot and the environment where the robot navigates. In the case of Micro-Aerial vehicle, in which the reduced size of the robot does not allow to embed many sensors onboard [27], [55], [56]; the use of synchronized environmental data might improve the localization and navigation capabilities [27]. Still, these data are not captured in the same computer and the use of the timestamp methodologies might not be reliable enough for fast control. The proposed methodology has been demonstrated enough reliable for the comparison of attitude data of an object on the space from different data sources.

\section{Acknowledgement:}

This research has been partially supported by a Grant by STMicroelectronics, which also provided the core sensors and the microcontroller. This work was also supported in part by: the Global COE Program "Global Robot Academia", MEXT, Japan; the Advancement of University Education Project of Chinese government Grant \# [2007] - 3020; the JSPS Grant-in-aid for Scientific Research \#19700389; the Waseda University Grant for Special Research Projects (No. 266740); and the JSPS Postdoctoral Fellowship Program for Foreigner Researcher FY2008, Consolidated Research Institute for Advanced Science and Medical Care, Waseda University (ASMeW),. The authors would like to express their thanks to the Italian Ministry of Foreign Affairs, General Directorate for Cultural Promotion and Cooperation, for its support to RoboCasa. The authors would also like to express their gratitude to Life Performance Research, Okino Industries LTD, Japan ROBOTECH LTD, SolidWorks Corp, Dyden, for their support to the research.

\section{References:}

[1] Y. Masuda, M. Sekimoto, M. Nambu, Y. Higashi, T. Fujimoto, K. Chihara, and Y. Tamura, "An unconstrained monitoring system for home rehabilitation" Engineering in Medicine and Biology Magazine, IEEE, vol. 24, pp. 43-47, 2005.

[2] B. P. Jarochowski, SeungJung Shin, DaeHyun Ryu, and HyungJun Kim, "Ubiquitous Rehabilitation Center: An Implementation of a Wireless Sensor Network Based Rehabilitation Management System" International Conference on Convergence Information Technology, pp. 2349-2358, 2007.

[3] S. Bhardwaj, D.-S. Lee, S. C. Mukhopadhyay, and W.-Y. Chung, "Ubiquitous Healthcare Data Analysis and Monitoring Using Multiple 
Wireless Sensors for Elderly Person" Sensor \& Transducer Journal, vol. 90, pp. 87-99, 2008.

[4] H. Lee, Y.-T. Kim, J.-W. Jung, K.-H. Park, D.-J. Kim, and Z. Z. Bien, "A 24-hour health monitoring system in a smart house" Gerontechnology, vol. 7, pp. 22-35, 2008.

[5] Nintendo, "Wii" [Online]. Available: http://wii.com.

[6] Microsoft, "Kinect - Xbox.com" [Online]. Available: http://www.xbox.com/en-US/kinect.

[7] R. Antonio Benitez, T. Guillermo de los Santos, and R. Daniel Vallejo, "Forward Kinematics for Virtual Agents" Engineering Letters, vol. 15, 2007.

[8] Yisheng Chen, J. Lee, R. Parent, and R. Machiraju, "Markerless monocular motion capture using image features and physical constraints" in Computer Graphics International, pp. 36-43, 2005.

[9] M. Zecca, F. Cavallo, M. Saito, N. Endo, Y. Mizoguchi, S. Sinigaglia, K. Itoh, H. Takanobu, G. Megali, O. Tonet, P. Dario, A. Pietrabissa, and A. Takanishi, "Analysis of the Surgeon's Performance during Laparoscopy by Using the Bioinstrumentation System WB-1R- towards the development of a Global Performance Index -" in IEEE/RSJ International Conference on Intelligent Robots and Systems (IROS), vol. Analysis and Control of Medical Robots I - TuD7, pp. 1272-1277, 2007.

[10] "Aurora Electromagnetic Measurement System" [Online]. Available: http://www.ndigital.com/medical/aurora.php.

[11] D. Roetenberg, P. Slycke, A. Ventevogel, and P. H. Veltink, "A portable magnetic position and orientation tracker" Sensors and Actuators A: Physical, vol. 135, pp. 426-432, 2007.

[12] D. Vlasic, R. Adelsberger, G. Vannucci, J. Barnwell, M. Gross, W. Matusik, and J. Popović, "Practical motion capture in everyday surroundings" $A C M$ Trans. Graph., vol. 26, no. 3, p. 35, 2007.

[13] G. Venture, K. Yamane, Y. Nakamura, and T. Yamamoto, "Identification of Human Limb Viscoelasticity using Robotics Methods to Support the Diagnosis of Neuromuscular Diseases" Int. J. Rob. Res., vol. 28, no. 10, pp. 1322-1333, 2009.

[14] Y. Nakamura, K. Yamane, Y. Fujita, and I. Suzuki, "Somatosensory computation for man-machine interface from motion-capture data and musculoskeletal human model" IEEE Transactions on Robotics, vol. 21, no. 1, pp. 58-66, 2005.

[15] "Vicon Systems," 2009. [Online]. Available: http://www.vivometrics.com/.

[16] S. Scapellato, F. Cavallo, C. Martelloni, and A. M. Sabatini, "In-use calibration of body-mounted gyroscopes for applications in gait analysis" Sensors and Actuators, vol. 123, pp. 418-422, 2005.

[17] T. Harada, T. Gyota, Y. Kuniyoshi, and T. Sato, "Development of Wireless Networked Tiny Orientation Device for Wearable Motion Capture and Measurement of Walking Around, Walking Up and Down, and Jumping Tasks" in IEEE/RSJ International Conference on Intelligent Robots and Systems, , pp. 4135- 4140, 2007.

[18] E. R. Bachmann, Xiaoping Yun, D. McKinney, R. B. McGhee, and M. J. Zyda, "Design and implementation of MARG sensors for 3-DOF orientation measurement of rigid bodies" in IEEE International Conference on Robotics and Automation, , vol. 1, pp. 1171-1178, 2003.

[19] Y. Mizoguchi, K. Itoh, M. Saito, N. Endo, M. Zecca, H. Takanobu, and A. Takanishi, "Development of a Bioinstrumentation System for Interaction 
with a Robot - Motion Capture System of Upper Body using Small Attitude Sensor Modules-" in 25th Annual Conference of the Robotics Society of Japan (RSJ2007), p. 2012, 2007.

[20] E. Foxlin, "Inertial head-tracker sensor fusion by a complementary separatebias Kalman filter", Proceedings of the IEEE Virtual Reality Annual International Symposium, pp. 185-194, 267, 1996.

[21] E. Foxlin, "Motion tracking requirements and technologies" in Handbook of Virtual Environment Technologies, Hillsdale, NJ: Lawrence Erlbaum, pp. 163-210, 2002.

[22] D. Roetenberg, P. J. Slycke, and P. H. Veltink, "Ambulatory Position and Orientation Tracking Fusing Magnetic and Inertial Sensing", IEEE Transactions on Biomedical Engineering, vol. 54, no. 5, pp. 883-890, 2007.

[23] S. You and U. Neumann, "Fusion of vision and gyro tracking for robust augmented reality registration", Proceedings Virtual Reality, pp. 71-78, 2001.

[24] J. Hightower and G. Borriello, "Particle filters for location estimation in ubiquitous computing: A case study", Proceedings of international Conference on Ubiquitous Computing (UBICOMP), p. 88-106, 2004.

[25] J. J. LaViola, “A comparison of unscented and extended Kalman filtering for estimating quaternion motion" in American Control Conference, vol. 3, pp. 2435-2440, 2003.

[26] D. B. Kingston and R. W. Beard, "Real-time attitude and position estimation for small UAVs using low-cost sensors" in AIAA 3rd Unmanned Unlimited Systems Conference and Workshop, 2004.

[27] G. H. Lee, M. Achtelik, F. Fraundorfer, M. Pollefeys, and R. Siegwart, "A benchmarking tool for MAV visual pose estimation", 11th International Conference on Control Automation Robotics \& Vision (ICARCV), pp. 15411546, 2010.

[28] Z. Lin, M. Zecca, S. Sessa, L. Bartolomeo, H. Ishii, K. Itoh, and A. Takanishi, "Development of an ultra-miniaturized inertial measurement unit WB-3 for human body motion tracking", IEEE/SICE International Symposium on System Integration (SII), pp. 414-419, 2010.

[29] E. Foxlin and Y. Altshuler, "Motion-tracking" U.S. Patent 647415905-Nov2002.

[30] S. Sessa, M. Zecca, Z. Lin, L. Bartolomeo, K. Itoh, H. Ishii, Y. Mukaeda, Y. Suzuki, and A. Takanishi, "Ultra-miniaturized WB-3 Inertial Measurement Unit: Performance evaluation of the attitude estimation", IEEE International Conference on Robotics and Biomimetics (ROBIO), pp. 998-1003, 2010.

[31] M. Windolf, N. Götzen, and M. Morlock, "Systematic accuracy and precision analysis of video motion capturing systems exemplified on the Vicon-460 system" Journal of Biomechanics, vol. 41, no. 12, pp. 27762780, 2008.

[32] J. J. Craig, "Introduction to Robotics: Mechanics and Control", 3rd ed. Prentice Hall, 2004.

[33] H. Zhou, H. Hu, and Y. Tao, "Inertial measurements of upper limb motion" Medical and Biological Engineering and Computing, vol. 44, no. 6, pp. 479487, 2006.

[34] D. G. Lloyd, J. Alderson, and B. C. Elliott, "An upper limb kinematic model for the examination of cricket bowling: A case study of Mutiah Muralitharan" Journal of Sports Sciences, vol. 18, no. 12, pp. 975-982, 2000. 
[35] H. Alan, "Determinants of the gait transition speed during human locomotion: Kinematic factors" Journal of Biomechanics, vol. 28, no. 6, pp. 669-677, 1995.

[36] K. Tong and M. H. Granat, "A practical gait analysis system using gyroscopes" Medical Engineering \& Physics, vol. 21, no. 2, pp. 87-94, 1999.

[37] M. Zecca, S. Sessa, Z. Lin, T. Sasaki, T. Suzuki, K. Itoh, H. Iseki, and A. Takanishi, "Development of an Ultra-Miniaturized Inertial Measurement Unit for Objective Skill Analysis and Assessment in Neurosurgery: preliminary results" MICCAI 2009, Part I, Lecture Notes in Computer Science 5761, vol. 5671, pp. 443-500, 2009.

[38] S. Sessa, M. Zecca, Z. Lin, T. Sasaki, K. Itoh, and A. Takanishi, "Waseda Bioinstrumentation System 3 as a tool for objective rehabilitation measurement and assessment - development of the inertial measurement unit _", IEEE International Conference on Rehabilitation Robotics, pp. 115-120, 2009.

[39] Z. Lin, M. Zecca, S. Sessa, H. Ishii, and A. Takanishi, "Development of an Ultra-Miniaturized Inertial Measurement Unit for Jaw Movement Analysis during Free Chewing” Journal of Computer Science, vol. 6, no. 8, pp. 896903, 2010.

[40] S. Sessa, M. Zecca, Z. Lin, T. Sasaki, T. Suzuki, K. Itoh, H. Iseki, and A. Takanishi, "Objective skill analysis and assessment of neurosurgery by using the waseda bioinstrumentation system WB-3" in IEEE/RSJ International Conference on Intelligent Robots and Systems, pp. 4086-4091, 2009.

[41] G. Welch and G. Bishop, "An Introduction to the Kalman Filter" University of north Carolina at Chapel Hill, 1995.

[42] A. Kourepenis, J. Borenstein, J. Connelly, R. Elliott, P. Ward, and M. Weinberg, "Performance of MEMS inertial sensors", Position Location and Navigation Symposium, pp. 1-8, 1998.

[43] A. M. Sabatini, "Quaternion-based extended Kalman filter for determining orientation by inertial and magnetic sensing", IEEE Transactions on Biomedical Engineering, vol. 53, no. 7, pp. 1346-1356, 2006.

[44] A. M. Sabatini, "A wavelet-based bootstrap method applied to inertial sensor stochastic error modelling using the Allan variance" Meas. Sci. Technol., vol. 17, pp. 2980-2988, 2006.

[45] R. Alonso and M. D. Shuster, "Attitude-independent magnetometer-bias determination: A survey" Journal of the Astronautical Sciences, vol. 50, no. 4, pp. 453-475, 2002.

[46] D. Gebre-egziabher, G. H. Elkaim, J. D. Powell, and B. W. Parkinson, “A non-linear, two-step estimation algorithm for calibrating solid-state strapdown magnetometers", 8th International St. Petersburg confernce on Navigation Systems, p. 28-30, 2001.

[47] D. Gebre-Egziabher, G. H. Elkaim, J. D. Powell, and B. W. Parkinson, "Calibration of Strapdown Magnetometers in Magnetic Field Domain" J. Aerosp. Engrg., vol. 19, no. 2, pp. 87-102, 2006.

[48] D. Campolo, M. Fabris, G. Cavallo, D. Accoto, F. Keller, and E. Guglielmelli "A Novel Procedure for In-field Calibration of Sourceless Inertial/Magnetic Orientation Tracking Wearable Devices" The First IEEE/RAS-EMBS International Conference on Biomedical Robotics and Biomechatronics, pp. 471-476, 2006 
[49] R. E. Mayagoitia, A. V. Nene, and P. H. Veltink, "Accelerometer and rate gyroscope measurement of kinematics: an inexpensive alternative to optical motion analysis systems" Journal of Biomechanics, vol. 35, no. 4, pp. 537$542,2002$.

[50] L. Ojeda and J. Borenstein, "Non-GPS Navigation for Security Personnel and First Responders" The Journal of Navigation, vol. 60, no. 03, pp. 391407, 2007.

[51] J. J. Kavanagh and H. B. Menz, "Accelerometry: a technique for quantifying movement patterns during walking" Gait Posture, vol. 28, no. 1, pp. 1-15, 2008.

[52] F. Sivrikaya and B. Yener, "Time synchronization in sensor networks: a survey" IEEE Network, vol. 18, no. 4, pp. 45-50, Jul. 2004.

[53] D. Sankoff and J. B. Kruskal, "Time warps, string edits, and macromolecules: The theory and practice of sequence comparison", Addison-Wesley Publishing Company, Inc., 1983.

[54] S. Salvador and P. Chan, "Toward accurate dynamic time warping in linear time and space" Intelligent Data Analysis, vol. 11, no. 5, pp. 561-580, 2007.

[55] Shaojie Shen, N. Michael, and V. Kumar, "Autonomous multi-floor indoor navigation with a computationally constrained MAV" in IEEE International Conference on Robotics and Automation, pp. 20-25, 2011.

[56] L. Heng, L. Meier, P. Tanskanen, F. Fraundorfer, and M. Pollefeys, "Autonomous obstacle avoidance and maneuvering on a vision-guided MAV using on-board processing" in IEEE International Conference on Robotics and Automation, pp. 2472-2477, 2011. 